\title{
The Evaporation of Liquid Micro-Drops on the Heated Substrate
}

\author{
Andrey Semenov ${ }^{1,2,3 *}$ \\ ${ }^{1}$ Kutateladze Institute of Thermophysics SB RAS, 630090 Novosibirsk, Russia \\ ${ }^{2}$ Novosibirsk State University, 630090 Novosibirsk, Russia \\ ${ }^{3}$ National Research Tomsk Polytechnic University, 634050 Tomsk, Russia
}

\begin{abstract}
Evaporation of a heated sessile water micro-drop was studied experimentally at the substrate temperature and surrounding atmosphere from 30 to $50{ }^{\circ} \mathrm{C}$. The studies were performed on the float glass substrate with aluminum nanocoating of optical quality. The research has shown that the specific rate of evaporation (mass loss per unit of the drop surface area) increases with the decrease in droplet volume and at the last stage several times exceeds the initial value.
\end{abstract}

\section{Introduction}

Some of the effective solutions for removal of high heat flux densities are spray cooling systems [1] and systems using the stratified two-phase flow in a microchannel $[2,3]$. In certain regimes in these systems the heat is removed due to intense evaporation of liquid micro-drops. In the literature, the evaporation of liquid drops on the heated plate was, as a rule, studied using sufficiently large droplets with an initial volume of about $100 \mu 1$ [4-9]. This paper investigates the evaporation of liquid droplets having an initial volume of less than $1 \mu$ l.

\section{Experimental setup and methods}

A liquid drop is placed on the surface with a known wettability. Then, the droplet is heated up to the onset of quasistationary heat transfer between the solid substrate and the liquid drop. The used working fluid is distilled deionized nanofiltered water of Milli-Q company. The use of water with a relatively high boiling point as working fluid provides for a rather detailed investigation of the influence of temperature difference between the solid surface and the surrounding atmosphere on the intensity of evaporation and heat transfer. The temperature difference between the solid surface and the atmosphere varies from 30 to 50 ${ }^{0} \mathrm{C}$.

The experiments on water drops evaporation in the open atmosphere were made on the experimental stand whose scheme is presented in Fig. 1. The work area was an aluminum

\footnotetext{
* Corresponding author: $\underline{\text { semenov.itp@gmail.com }}$
} 
plate of $15 \mathrm{~mm}$ thickness, on whose bottom side the heater was located. The used heater was a Peltier element with dimensions $40 \times 40 \mathrm{~mm} 2$. On the upper side of the aluminum plate the investigated substrate was fixed. It consisted of a disk with a diameter of $52 \mathrm{~mm}$ and a thickness of $3.2 \mathrm{~mm}$. Measurement of the surface temperature of the studied substrate was carried out using a flat thermocouple, fixed at a distance of 1-2 $\mathrm{mm}$ from the contact line of the liquid drop. The temperature under the substrate was measured by three thermocouples of K-type. Thermocouples were placed radially at different distances from the center of the disc and served to measure the temperature distribution along the substrate radius. The working surface was made of float glass with aluminum nanocoating of optical quality. The relative humidity of air in the room during the experiments was $20-30 \%$.

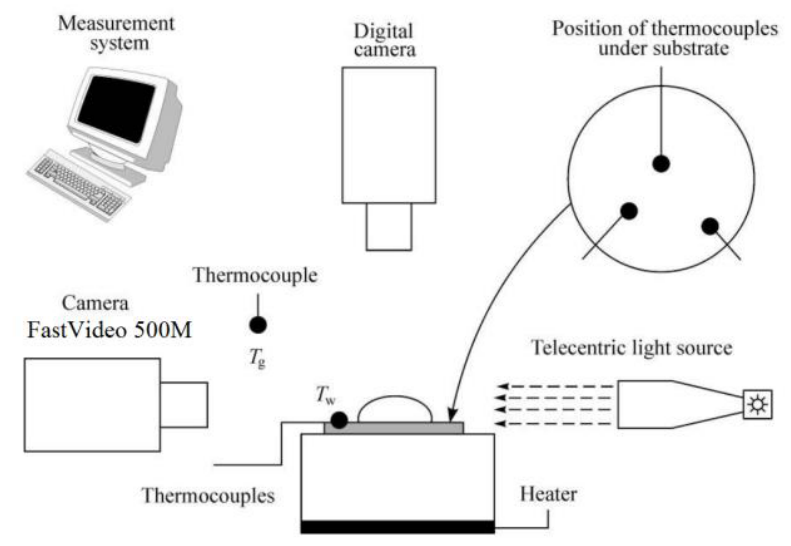

Fig. 1. Scheme of experimental stand.

The authors used two optical techniques. The first one is a shadow method, consisting of the halogen light source Edmunds Optics MI-150, connected by a fiberoptic lightguide with a system of lenses of Edmunds Optics Telecentric backlight illuminator. This system generates a plane-parallel light beam of $50 \mathrm{~mm}$ diameter at the output of one side of the work area. High-speed video camera FastVideo 500M with a microscope lens 10X Mitutoyo Plan Apo Infinity-Corrected Long WD, connected through the focusing bellow Novoflex Balpro TS, on the other side of the droplet. The spatial resolution of this system was $1 \mu \mathrm{m} /$ pixel, and the frequency of shooting was up to 500 frames per second. Using the shadow method we obtained shadow images of the liquid drop profile. The images were processed by the Drop Shape Analysis software of KRUSS company. Shadow photography was used to select the drop profile by the method of Young-Laplace. Further, the following geometric parameters of the liquid drop were determined: the base diameter, maximum diameter, height, volume, contact angles (right, left, middle), the surface area, and the basal area.

The second optical technique consisted of a digital video camera Point Grey Grasshopper3 (4.1 Mpix) with a microscope lens 20X Mitutoyo Telecentric Objective. It was placed over the liquid drop and allowed visualizing the evaporation from the top and controlling the symmetry of the droplet. Spatial resolution of the system was $0.5 \mu \mathrm{m} / \mathrm{pixel}$, and the frequency of shooting was up to 90 frames per second. If the drop lost its symmetry, the experiment repeated.

\section{Results}

The experiment began with a preliminary preparation of the investigated substrate, which consisted in a thorough cleaning of the working surface. Then the whole substrate 
was placed in a reservoir with distilled water for a day. Thereafter, to remove residual water from the surface the substrate was flushed with clean compressed air designed for cleaning optical components. A liquid drop of preset size was placed on the surface, heated to the desired temperature using a high-precision dispenser Lenpipet. The lens used in the shadow method was focused (depth of field was a few $\mathrm{mm}$ ); and after that the periodic shooting was performed until complete evaporation of the liquid droplet (Fig.2). The upper chamber was switched on simultaneously with the shadow method.

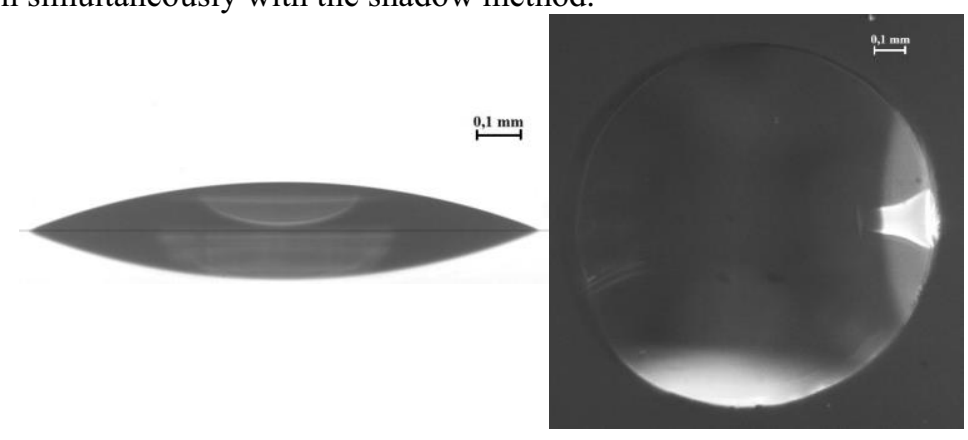

Fig. 2. Images of liquid droplets from the side and from above, $V=0.06 \mu 1, \mathrm{~T}_{\mathrm{s}}=40^{\circ} \mathrm{C}$.

The dependence of the specific rate of liquid evaporation on time at different initial surface temperature is shown in Fig. 3. The evaporation rate was calculated as a loss in the droplet mass per unit of droplet surface area per unit of time. All built-in data were synchronized to the time of complete evaporation of liquid droplets. The research has shown that the specific rate of evaporation increases with the decrease in droplet volume and at the last stage several times exceeds the initial value. The obtained results are in good agreement with the results of $[5,6]$, studying the evaporation of drops with initial volume of about $100 \mu 1$.

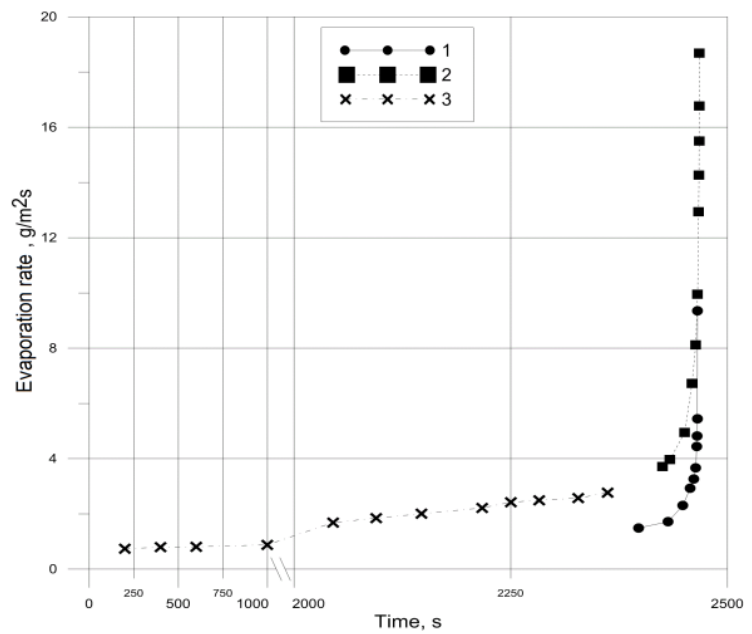

Fig. 3. The dependence of specific evaporation rate on time. 1 - data obtained with the substrate temperature $\mathrm{T}_{\mathrm{s}}=30^{\circ} \mathrm{C}$, the initial drop volume $\mathrm{V}_{0}=0.22 \mu \mathrm{l} ; 2-\mathrm{T}_{\mathrm{s}}=45^{\circ} \mathrm{C}, \mathrm{V}_{0}=95.16 \mu \mathrm{l} ; 3$ - data obtained in the work [5] at $\mathrm{T}_{\mathrm{s}}=45^{\circ} \mathrm{C}, \mathrm{V}_{0}=95.16 \mu \mathrm{l}$.

The study was supported by the grant of the Russian Science Foundation (agreement No. 14-19-01755). 


\section{References}

1. J. Kim, Int. J. Heat Fluid Fl. 28, 753 (2006)

2. O.A. Kabov, Yu.V. Lyulin, I.V. Marchuk and D.V. Zaitsev, Int. J. Heat Fluid Fl. 28, 103 (2007)

3. D.V. Zaitsev, D.A. Rodionov and O.A. Kabov, Tech. Phys. Lett. 35, 680 (2009)

4. O.A. Kabov and D.V. Zaitsev, Doklady Physics 58, 292 (2013)

5. E.Ya. Gatapova, A.A. Semenov, D.V. Zaitsev, O.A. Kabov, Colloid Surf. A 441, 776 (2014)

6. A.A. Semenov, D.V. Feoktistov, D.V. Zaitsev, G.V. Kuznetsov, and O.A. Kabov, Thermophys. Aeromech. 22, 771 (2015)

7. I.V. Marchuk, A. Karchevsky, A. Surtaev, and O.A. Kabov, Int. J. Aerospace Eng. 2015, 5 (2015)

8. G. V. Kuznetsov, D.V. Feoktistov, E. G. Orlova, Thermophys. Aeromech. 23, 17 (2016)

9. G.V. Kuznetsov, D.V. Feoktistov, E.G. Orlova, K.A. Batishcheva, Colloid J. 78, 335 (2016) 